Research Paper

\title{
Prognostic value of downregulated 5-hydroxymethyl- cytosine expression in renal cell carcinoma: a 10 year follow-up retrospective study
}

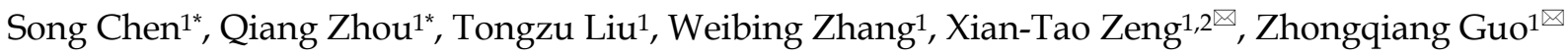 \\ 1. Department of Urology, Zhongnan Hospital of Wuhan University, Wuhan 430071, China. \\ 2. Center for Evidence-Based and Translational Medicine, Zhongnan Hospital of Wuhan University, Wuhan 430071, China. \\ "These authors contributed equally to this work.
}

$\triangle$ Corresponding author: Dr. Zhongqiang Guo, Email: guozhongqiang@whu.edu.cn, Tel. +86-27-6781-3104, Fax: +86-27-6781-2892; and Dr. Xian-Tao Zeng, Email: zengxiantao1128@163.com, Tel. +86-27-6781-3104, Fax: +86-27-6781-2892.

(c) The author(s). This is an open access article distributed under the terms of the Creative Commons Attribution License (https://creativecommons.org/licenses/by/4.0/). See http://ivyspring.com/terms for full terms and conditions.

Received: 2019.07.09; Accepted: 2019.11.02; Published: 2020.01.01

\begin{abstract}
5-hydroxymethylcytosine $(5 \mathrm{hmC})$ is converted from DNA methylation of cytosine $(5 \mathrm{mC})$ by the catalysis of TET proteins, and proposed to be involved in tumorigenesis. However, the prognostic value of $5 \mathrm{hmC}$ in renal cell carcinoma (RCC) is still unclear. This study aimed to define the clinical significance of $5 \mathrm{hmC}$ in RCC. We performed dot blot assays to measure the relative expression of $5 \mathrm{hmC}$ in RCC. We reviewed the clinical records of $310 \mathrm{RCC}$ patients and performed immunohistochemical $(\mathrm{IHC})$ staining of $5 \mathrm{hmC}$. The overall survival (OS) and cancer specific survival (CSS) of all patients were recorded over a 10-year follow-up period. Effective prognostic nomograms which contained $5 \mathrm{hmC}$ were established to provide individualized OS and CSS in RCC. $5 \mathrm{hmC}$ expression level was significantly decreased in RCC tissues compared with those in the normal counterparts. Kaplan-Meier curves revealed that high $5 \mathrm{hmC}$ expression had a good prognostic impact on RCC patients. Cox multivariate survival analyses further indicated $5 \mathrm{hmC}$ was an independent prognostic factor for RCC survival. Nomograms constructed based on cox regression analysis were available to calculate the survival probability directly. Calibration curves displayed good agreements. The findings were validated with an independent external cohort included 77 RCC cases. Thus, we believe we have found a significative prognostic factor for RCC.
\end{abstract}

Key words: renal cell carcinoma, 5-hydroxymethylcytosine, clinical significance, nomogram.

\section{Introduction}

Renal cell carcinoma (RCC) is a malignant tumor mainly originating from renal tubular epithelial cells, accounting for $2-3 \%$ of adult malignant tumors. Currently, the incidence rate and the mortality rate are increasing in worldwide [1-2]. Patients with early stage RCC have no obvious clinical symptoms, and they often have progressed to the advanced stage when obvious discomfort occurs. More specifically, RCC is a common group of chemotherapy-resistant diseases, which are not sensitive to traditional chemoradiotherapy or hormone therapy [3-4]. At present, the most common treatment for RCC is radical nephrectomy or nephron sparing partial nephrectomy. However, about $30 \%$ of patients have metastasized cancer when diagnosed, and about half of the patients who have not metastasized will experience recurrence or metastasis after radical resection [5-6].

The clear cell renal cell carcinoma (ccRCC) is the primary type of RCC, which represents $75 \%-80 \%$ of all RCCs [7]. Some studies have shown that patients with ccRCC have a poor prognosis. The 5 -year survival rate of ccRCC patients is about $60 \%$, which is lower than other types of RCC patients [8-9]. Currently, there are 
many clinical factors used to evaluate the prognosis of RCC, including histological type, Fuhrman's nuclear grade, tumor stage, tumor necrosis, lymph node metastasis and vein invasion. However, a single clinical factor is not ideal for predicting the prognosis of RCC [10-11]. Therefore, prognostic monitoring of RCC and more effective targeted therapies are essential to reduce patient mortality and improve cure rate.

There are exact evidences that the Von Hippel-Lindau (VHL) tumor suppressor gene is mutated or inactivated in more than $80 \%$ of ccRCC patients. However, in mouse (Mus musculus) experiments, it was found that the deletion of this gene did not cause the formation of clear cell carcinoma, which indicates that there are other tumorigenic mechanisms [12-13]. Previous studies have also found that mutations associated with renal carcinogenesis and prognosis occur mainly in genes encoding epiregulatory factors, such as the BAP1 gene that regulates histone $\mathrm{H} 2 \mathrm{~A}$ ubiquitination, the histone methyltransferase gene SETD2, and the TET2 gene which catalyzes the conversion of DNA methylation of cytosine $(5 \mathrm{mC})$ to $5 \mathrm{hmC}[14-15]$.

As one of the most widely studied epigenetic modifications, $5 \mathrm{mC}$ of the $\mathrm{CpG}$ dinucleotide in gene promoter is usually related to the transcriptional silencing of cancer cells [16]. Several important studies showed DNA methyltransferases could catalyze and maintain the transformation of cytosine to methyl cytosine, but the Ten-Eleven-Translocation (TET) family of TET1, TET2 and TET3 mediate the reverse process [17-19]. Gradually, these TET proteins mediate the transformation of $5 \mathrm{mC}$ to $5 \mathrm{hmC}$, 5 -formyl cytosine and 5-carboxyl cytosine [20].

Emerging evidence indicates that $5 \mathrm{hmC}$ may also act a role of stable epigenetic marker with incomplete characterization [21]. In our previous study, we found that almost all patients with RCC have a loss of $5 \mathrm{hmC}$, which is considered to be a result of the downregulation of isocitrate dehydrogenase 1 (IDH1) [22]. Nevertheless, the prognostic value of $5 \mathrm{hmC}$ in RCC is still unclear. In present study, we attempted to define the clinical significance of $5 \mathrm{hmC}$ in RCC (including ccRCC and other RCC).

\section{Material and methods}

\section{Study Patients}

This study contained a development cohort and a validation cohort. The development cohort included 310 RCC patients at the Department of Urology, Zhongnan Hospital of Wuhan University from January 2007 to March 2015. All patients had undergone surgery treatment meanwhile surgical tissue specimens was gathered. Dot blot assays and IHC staining of $5 \mathrm{hmC}$ were performed in these surgical tissue specimens. Clinical, pathological, follow-up data record was collected. The analyzed clinical and pathological data consisted of age, gender, tumor size, capsule invasion, vein invasion, tumor necrosis, TNM stage, clinical stage, Furhman grade and $5 \mathrm{hmC}$ level. The follow-up data included OS and CSS of RCC. All patients provided the informed consent. The Ethics Committee at Zhongnan Hospital of Wuhan University has approved the using clinical information and surgical tissue specimens in our study (approval number: 2015029). All procedures and ethical standards were done in accordance with the national research committee and/or institutional. The validation cohort was a tissue microarrays (TMA, ID: KIC387) purchased from Guilin Fanpu Biotech Co., Ltd, which included 77 RCC patients from July 2006 to October 2009.

\section{Inclusion criteria}

Patients were enrolled in this study if they met all the following criteria: (i) presence of primary RCC; (ii) without any a prior history of preoperative target therapy, chemoradiotherapy or hormone therapy; (iii) underwent radical nephrectomy or nephron sparing partial nephrectomy; (iv) surgical tissue specimens (tumor/normal) were collected; (v) had a complete and detailed clinical, pathological, follow-up data record.

\section{Exclusion criteria}

Patients meeting any of the following criteria were excluded: (i) presence of metastatic/secondary tumor of the kidney; (ii) any prior history of preoperative targeted therapy, chemoradiotherapy or hormone therapy; (iii) patients who did not undergo surgery; (iv) surgical tissue specimens were unavailable; (v) any incomplete clinical, pathological or follow-up data.

\section{Cell lines}

RCC cell lines (Ketr-3, OS-RC-2, 769-P and 786-O) were cultured in RPMI1640 medium (Gibco, China) supplemented with 10\% FBS. Human renal proximal tubular epithelial cell line (HK-2) was maintained in KSF medium with epidermal growth factor as well as bovine pituitary extract (Gibco, Carlsbad, CA, USA). All cell lines were purchased from the Stem Cell Bank, Chinese Academy of Sciences in Shanghai, China. These cell lines were grown at $5 \% \mathrm{CO}_{2}, 37^{\circ} \mathrm{C}$ in a humidified incubator (Thermo Scientific).

\section{Cell lines and tissue genomic DNA extraction}

A proportion of the surgical tissue specimens 
were cryopreserved at $-80^{\circ} \mathrm{C}$. We used tumor as well as the matched normal tissue from the same radical nephrectomy patients for DNA analysis. Genomic DNA was isolated from cultured RCC cell lines and surgical tissue specimens with the Qiagen DNA Mini Kit (250) (Qiagen, Cat\#: 51306).

\section{Quantitative $5 \mathrm{hmC}$ analysis}

Quantitative 5hmC analysis performed via DNA dot blotting. Genomic DNA was heated for deformation and then chilled; temperature/time were $95^{\circ} \mathrm{C} / 10 \mathrm{~min}$ and $4^{\circ} \mathrm{C} / 5 \mathrm{~min}$, respectively. The DNA samples were dried on wet Hybrid Membrane. The samples were then spotted on positively charged membranes and afterwards serially diluted in $\mathrm{NaOH} /$ EDTA solution. The membrane was blocked with 5\% non-fat milk in TBST for $1 \mathrm{~h}$ at room temperature (RT), followed by incubation with primary antibody against $5 \mathrm{hmC}$ (dilution 1:5000, Active motif) overnight at $4^{\circ} \mathrm{C}$. Anti-rabbit IgG-HRP antibody was incubated with the membrane for $1 \mathrm{~h}$ at RT. At last, the membrane was treated with ECL Kit (GE Amersham, Cat\#: RPN2232) after washing three times with TBST.

\section{Immunohistochemical staining and scoring}

A proportion of surgical tissue specimens were fixed with formalin to for paraffin-embedded. IHC analyses was performed on $4 \mu \mathrm{m}$ thick sections. Briefly, each slide was incubated with primary antibody against 5hmC (1: 5000, Active motif, Cat\#: 39999) overnight after a series of procedures (de-paraffin, antigen retrieval, rinse). This was followed by an incubation with the anti-rabbit IgG-HRP antibody (EnVision Dual Link, Dako) for 30 min. The membrane was then washed five times with TBST and enriched with the brown color of DAB Enhancer (Dako). The $5 \mathrm{hmC}$ expression was evaluated by three experienced pathologists. Receiver operating characteristic (ROC) curve was generated for $5 \mathrm{hmC}$ expression level to calculate the areas under the curve (AUC). The highest Youden's index, as the optimized point, was used to determine the optimal cut-off value of $5 \mathrm{hmC}$ expression level based on the ROC curve.

\section{Statistical analysis}

All continuous measures were compared by two-sample $t$ test, graded variables were analyzed with Mann-Whitney test. The associations between $5 \mathrm{hmC}$ expression level and specific clinicopathological factors in RCC patients (including ccRCC and other RCC) were analyzed with Chi-square test. Kaplan-Meier curves were generated to estimate OS and CSS, and the log-rank test was used to assess survival differences among subgroups. Cox univariate and multivariate survival analyses were used to estimate the independent factors of survival rate. Nomograms were generated based on cox regression analyses. The calibration curves were generated to assess the agreements of the nomogram-predicted probability with the actual observed probability. The stability (sensitivity and specificity) of the prediction nomograms were validated with the independent external cohort. We used SPSS 16.0 and GraphPad Prism 7 to perform all statistical analyses. Nomograms and calibration curves were generated with $R$ version 3.5.0 and a p value $<0.05$ was considered statistically significant.

\section{Results}

\section{$\mathbf{5 h m C}$ level was significantly decreased in RCC}

To detect the change of $5 \mathrm{hmC}$ level in RCC tumorigenesis, we performed DNA dot blot assay using RCC and normal counterparts. The results showed $5 \mathrm{hmC}$ was downregulated in 3 RCC tumor samples compared with the matched normal tissues. RCC cell lines also yielded similar results, in which human renal proximal tubular epithelial cell line (HK-2) expressed highest level of $5 \mathrm{hmC}$ compared with all four RCC cell lines (Figure 1A). In the following study, we performed IHC staining in RCC and normal kidney tissues adjacent to cancer. IHC staining presented that $5 \mathrm{hmC}$ level in $310 \mathrm{RCC}$ patients' tumor tissues was significantly downregulated as compared with that in 248 adjacent kidney tissues (Figure 1B, 1C). With the method mentioned, the cut-off value of $5 \mathrm{hmC}$ relative expression level was determined as $20.4 \%$ (AUC $=0.836$, Supplementary Figure S1). So " $5 \mathrm{hmC}$ low" and "5hmC high" represented cases in which $\leq 20 \%$ and $>20 \%$ cells were positive for IHC staining of $5 \mathrm{hmC}$, respectively (Figure 1D).

\section{Patient characteristics}

Of 310 RCC patients, those diagnosed with ccRCC were accounted for 230 (74.2\%). The median follow-up time was 90.9 months (range 0.3-122.4 months) for all patients. Additionally, 215 (69.4\%) patients were followed up for more than 5 years, and 111 (35.8\%) patients more than 10 years. During follow-up, $135(43.5 \%)$ patients died and the 5-year and 10-year OS rates were $70.6 \%$ and $56.5 \%$, respectively. Table 1 listed the clinicopathological parameters (gender, age, tumor size, capsule invasion, vein invasion, tumor necrosis, TNM stage, clinical stage, Furhman grade and 5hmC level). Briefly, 114 female and 196 male patients with a mean age of 61.8 y (25-87 y). 168 patients identified as were " $5 \mathrm{hmC}$ 
low" and 142 were "5hmC high". Two-sample $t$ test and standard nonparametric Mann-Whitney $U$-test showed ccRCC patients in $5 \mathrm{hmC}$ low group with a higher clinical stage, higher $\mathrm{T}$ stage, lower Furhman grade, compared with $5 \mathrm{hmC}$ high group RCC patients $(\mathrm{p}<0.05$, Table 1$)$.

Table 1. Clinical characteristics of $\mathrm{ccRCC}$ and other RCC patients.

\begin{tabular}{|c|c|c|c|c|}
\hline Variables & $\begin{array}{l}\text { RCC } \\
(n=310)\end{array}$ & $\begin{array}{l}\text { ccRCC } \\
(n=230)\end{array}$ & $\begin{array}{l}\text { other RCC } \\
(\mathrm{n}=80)\end{array}$ & $p$ value \\
\hline Age (years), n (\%) & & & & 0.156 \\
\hline Average/Median & $61.8 \pm 13.5 / 64$ & $61.3 \pm 13.5 / 63$ & $63.5 \pm 13.5 / 66$ & \\
\hline Range & $25-87$ & $25-87$ & $28-87$ & \\
\hline$<65$ & $161(51.9)$ & $125(54.3)$ & $36(45.0)$ & \\
\hline$\geq 65$ & $149(48.1)$ & $105(45.7)$ & $44(55.0)$ & \\
\hline Gender, n (\%) & & & & 0.234 \\
\hline Female & $114(36.8)$ & $89(38.7)$ & $25(31.2)$ & \\
\hline Male & $196(63.2)$ & $141(61.3)$ & $55(68.8)$ & \\
\hline Tumor Size (cm), n (\%) & & & & 0.771 \\
\hline Average/Median & $6.2 \pm 3.7 / 5.0$ & $6.1 \pm 3.6 / 5.0$ & $6.4 \pm 4.1 / 5.0$ & \\
\hline Range & $0.5-23.0$ & $0.9-23.0$ & $0.5-18.0$ & \\
\hline$\leq 5$ & 159 (51.3) & $115(50.0)$ & $44(55.0)$ & \\
\hline$>5$ & $151(48.7)$ & $115(50.0)$ & $36(45.0)$ & \\
\hline Capsule Invasion, $\mathrm{n}(\%)$ & & & & 0.097 \\
\hline No & $226(72.9)$ & $162(70.4)$ & $64(80.0)$ & \\
\hline Yes & $84(27.1)$ & $68(29.6)$ & $16(20.0)$ & \\
\hline Vein Invasion, $\mathbf{n}(\%)$ & & & & 0.187 \\
\hline No & $252(81.3)$ & $183(79.6)$ & $69(86.2)$ & \\
\hline Yes & $58(18.7)$ & $47(20.4)$ & $11(13.8)$ & \\
\hline Necrosis, $\mathbf{n}(\%)$ & & & & 0.044 \\
\hline No & $281(90.6)$ & $213(92.6)$ & $68(85.0)$ & \\
\hline Yes & $29(9.4)$ & $17(7.4)$ & $12(15.0)$ & \\
\hline T Stage, n (\%) & & & & 0.035 \\
\hline $\mathrm{T} 1$ & 179 (57.7) & $125(54.3)$ & $54(67.5)$ & \\
\hline $\mathrm{T} 2$ & $25(8.1)$ & $18(7.8)$ & $7(8.8)$ & \\
\hline T3 & $102(32.9)$ & $85(37.0)$ & $17(21.2)$ & \\
\hline $\mathrm{T} 4$ & $4(1.3)$ & $2(0.9)$ & $2(2.5)$ & \\
\hline N Stage, n (\%) & & & & 0.461 \\
\hline No/Nx & $298(96.1)$ & $220(95.7)$ & $78(97.5)$ & \\
\hline N1 & $6(1.9)$ & $5(2.2)$ & $1(1.3)$ & \\
\hline N2 & $6(1.9)$ & $5(2.2)$ & $1(1.3)$ & \\
\hline M Stage, n (\%) & & & & 0.871 \\
\hline $\mathrm{M} 0 / \mathrm{Mx}$ & $288(92.9)$ & $214(93.0)$ & $74(92.5)$ & \\
\hline M1 & $22(7.1)$ & $16(7.0)$ & $6(7.5)$ & \\
\hline Clinical Stage, $\mathbf{n}(\%)$ & & & & 0.026 \\
\hline 1 & $175(56.5)$ & $121(52.6)$ & $54(67.5)$ & \\
\hline 2 & $25(8.1)$ & $18(7.8)$ & $7(8.8)$ & \\
\hline 3 & $85(27.4)$ & $73(31.7)$ & $12(15.0)$ & \\
\hline 4 & $25(8.1)$ & $18(7.8)$ & $7(8.8)$ & \\
\hline Furhman Grade, n (\%) & & & & $<0.001$ \\
\hline 1 & $64(20.6)$ & $62(27.0)$ & $2(2.5)$ & \\
\hline 2 & $122(39.4)$ & $90(39.1)$ & $32(40.0)$ & \\
\hline 3 & $89(28.7)$ & $62(27.0)$ & $27(33.8)$ & \\
\hline 4 & 35 (11.3) & $16(7.0)$ & $19(23.8)$ & \\
\hline $5 \mathrm{hmC} \%, \mathrm{n}(\%)$ & & & & $<0.001$ \\
\hline Average/Median & $28.2 \pm 25.0 / 20$ & $23.8 \pm 21.3 / 20$ & $40.7 \pm 30.2 / 40$ & \\
\hline Range & $0-23.0$ & $0-90$ & $1-100$ & \\
\hline Low $(\leq 20 \%)$ & $168(54.2)$ & $140(60.9)$ & $28(35.0)$ & \\
\hline High $(>20 \%)$ & $142(45.8)$ & $90(39.1)$ & $52(75.0)$ & \\
\hline
\end{tabular}

Of 77 RCC patients in the validation cohort, 59 (76.6\%) patients were diagnosed with ccRCC. The median follow-up time was 82.1 months (range 1.1-133.6 months). The detailed clinical parameters of enrolled patients in development cohort and validation cohort were presented in Supplementary Table s1, there was no significant difference in clinical parameters between the two cohorts (all $\mathrm{p}>0.05$ ).

\section{Correlation analysis between $5 \mathrm{hmC}$ expression level and clinicopathological factors of patients with RCC}

The correlations between $5 \mathrm{hmC}$ and clinicopathological factors were analyzed to define the clinical significance of $5 \mathrm{hmC}$ in RCC. Chi-square testing showed that there were no significant correlations between $5 \mathrm{hmC}$ level and age, gender, tumor necrosis, $\mathrm{N}$ stage, or Furhman grade (Table 2). Remarkably, we found $5 \mathrm{hmC}$ level was significantly correlated with tumor size, capsule invasion, vein invasion, $T$ stage, $M$ stage and clinical stage with $\mathrm{p}$ values of $0.011,0.001,0.012,0.003,0.002,0.001$, respectively. Interestingly, these differences were mainly concentrated in other RCC and 5hmC did not seem to affect these clinicopathological variables in ccRCC patients (Table 2). In the validation cohort, chi-square testing also showed the consistent results, which validated our finding (Supplementary Table s2).

\section{Kaplan-Meier survival analysis between $5 \mathrm{hmC}$ expression level and patient survival}

In the Kaplan-Meier survival analyses, compared to those RCC patients with low 5hmC, patients with high $5 \mathrm{hmC}$ had increased OS and CSS (Log-rank, $p=0.0054, p<0.0001$, respectively, Figure $2 \mathrm{~A})$. This finding indicated that high $5 \mathrm{hmC}$ expression led to a good prognostic impact for RCC patients. Similar results were also yielded in ccRCC patients (Figure 2B) and other RCC patients (Figure 2C).

\section{Cox univariate and multivariate analyses of patient survival}

Cox univariate and multivariate survival analyses were performed to assess the prognostic value of $5 \mathrm{hmC}$ level for RCC and ccRCC patients. Univariate survival analysis showed age, tumor size, capsule invasion, vein invasion, TNM stage, clinical stage, Furhman grade and $5 \mathrm{hmC}$ level were factors significantly affecting RCC patient OS. Tumor size, capsule invasion, vein invasion, TNM stage, clinical stage, Furhman grade and $5 \mathrm{hmC}$ level were factors significantly affecting CSS. Moreover, multivariate survival analyses revealed that age (HR: 1.043; 95\% CI: 1.027-1.059; $\mathrm{p}<0.001$ ), capsule invasion (HR: 1.745; 95\% CI: 1.059-2.877; $\mathrm{p}=0.029), \mathrm{N}$ stage (HR: 3.110; 95\%CI: 1.491-6.485; $\mathrm{p}=0.002$ ), clinical stage (HR: 1.334; 95\%CI: 1.050-1.695; $\mathrm{p}=0.018$ ), Furhman grade (HR: 1.508; 95\%CI: 1.238-1.837; $\mathrm{p}<0.001)$ and $5 \mathrm{hmC}$ level 
(HR: $0.657 ; \quad 95 \% \mathrm{CI}: \quad 0.455-0.949 ; \quad \mathrm{p}=0.025$ ) were independent prognostic factors for RCC patient OS, but only clinical stage (HR: 2.381; 95\%CI: 1.892-2.996; $\mathrm{p}<0.001)$, Furhman grade (HR: 1.740; 95\%CI:
1.325-2.285; $\mathrm{p}<0.001$ ) and 5hmC level (HR: 0.404; 95\%CI: 0.231-0.709; $p=0.002)$ were the independent prognostic factors for RCC patient CSS (Table 3, Table 4 , respectively).

A
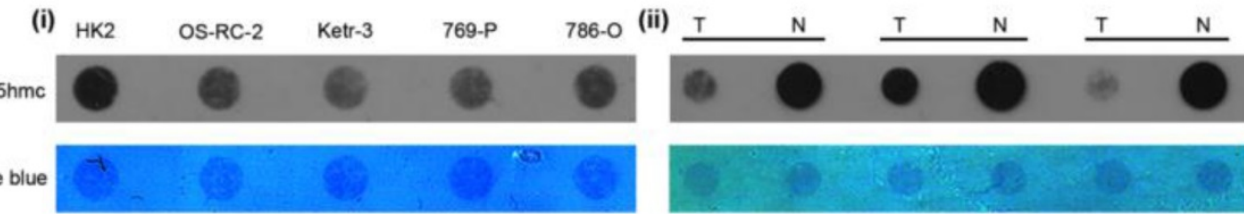

B
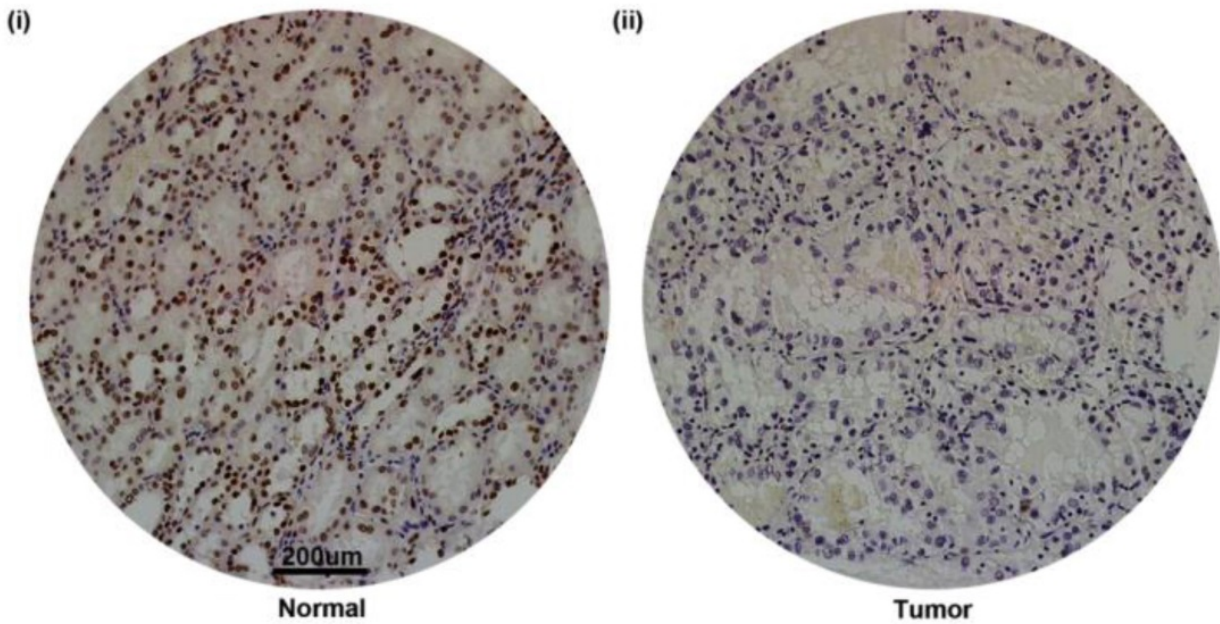

C

(i)

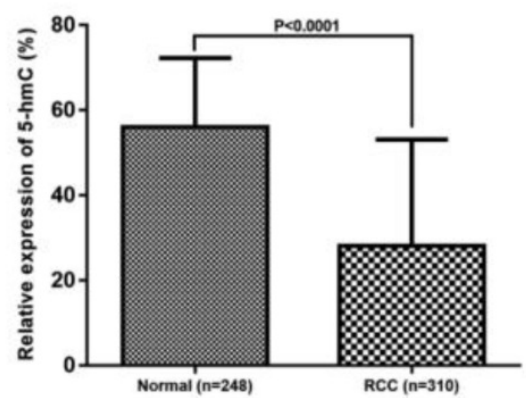

D (ii)

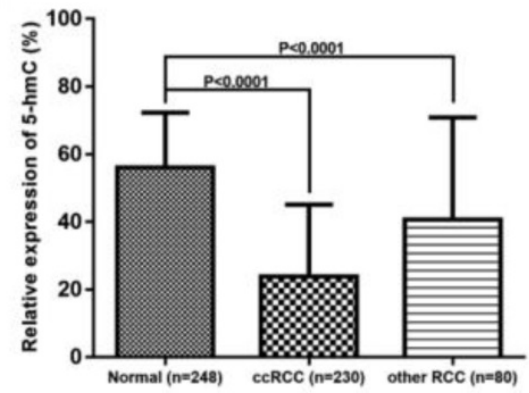

(ii)

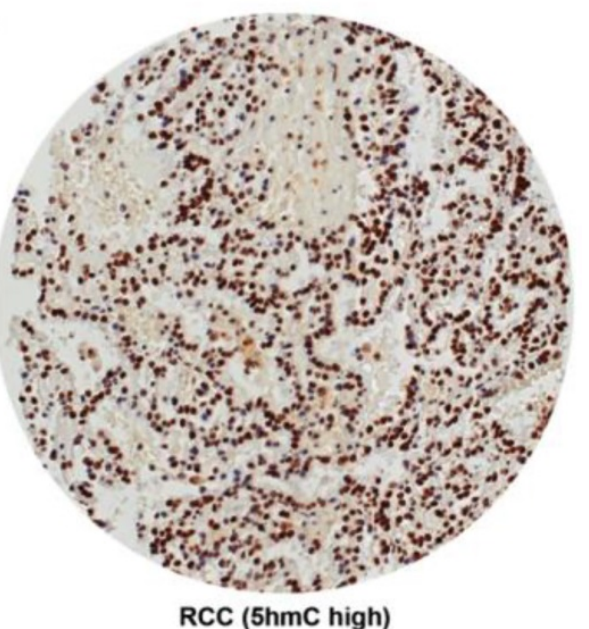

Figure 1. Relative expression of $5 \mathbf{h m C}$ in RCC (A) Dot blot assays of $5 \mathrm{hmC}$ in RCC cell lines/HK-2 as well as paired RCC tissues/renal tissues of three RCC patients. Equal loading was validated by methylene blue staining. T, tumor; $\mathrm{N}$, matched normal tissue; (B) A representative IHC staining of $5 \mathrm{hmC}$ in tumor and paired normal samples of RCC patients. Scare bar, $200 \mu \mathrm{m}$; (C) Relative expression of $5 \mathrm{hmC}$ level in RCC (include ccRCC and other RCC) tissues/renal tissues; (D) A representative IHC staining of " $5 \mathrm{hmC}$ low" RCC tissue and " $5 \mathrm{hmC}$ high" RCC tissue. " $5 \mathrm{hmC}$ low" and " $5 \mathrm{hmC}$ high" represented cases in which $\leq 20 \%$ and $>20 \%$ cells were positive for IHC staining of $5 \mathrm{hmC}$, respectively. Scare bar, $200 \mu \mathrm{m}$. 
Table 2. Correlations between $5 \mathrm{hmC}$ expression and clinicopathological factors of patients with RCC and ccRCC.

\begin{tabular}{|c|c|c|c|c|c|c|c|c|c|}
\hline \multirow[t]{2}{*}{ Characteristics } & \multicolumn{3}{|c|}{ 5hmC expression (RCC) } & \multicolumn{3}{|c|}{ 5hmC expression (ccRCC) } & \multicolumn{3}{|c|}{ 5hmC expression (other RCC) } \\
\hline & Low $(n=168)$ & High $(n=142)$ & $p$ & Low $(n=140)$ & $\operatorname{High}(n=90)$ & $p$ & Low $(n=28)$ & High $(n=52)$ & $p$ \\
\hline Age (years), $\mathrm{n}$ & & & 0.864 & & & 0.804 & & & 0.451 \\
\hline$<65$ & 88 & 73 & & 77 & 48 & & 11 & 25 & \\
\hline$\geq 65$ & 80 & 69 & & 63 & 42 & & 17 & 27 & \\
\hline Gender, n & & & 0.959 & & & 0.546 & & & 0.527 \\
\hline Female & 62 & 52 & & 52 & 37 & & 10 & 15 & \\
\hline Male & 106 & 90 & & 88 & 53 & & 18 & 37 & \\
\hline Tumor Size $(\mathrm{cm}), \mathrm{n}$ & & & 0.011 & & & 0.177 & & & 0.011 \\
\hline$\leq 5$ & 75 & 84 & & 65 & 50 & & 10 & 34 & \\
\hline$>5$ & 93 & 58 & & 75 & 40 & & 18 & 18 & \\
\hline Capsule Invasion, $\mathrm{n}$ & & & 0.001 & & & 0.285 & & & $<0.001$ \\
\hline No & 110 & 116 & & 95 & 67 & & 15 & 49 & \\
\hline Yes & 58 & 26 & & 45 & 23 & & 13 & 3 & \\
\hline Vein Invasion, $\mathrm{n}$ & & & 0.012 & & & 0.141 & & & 0.071 \\
\hline No & 128 & 124 & & 107 & 76 & & 21 & 48 & \\
\hline Yes & 40 & 18 & & 33 & 14 & & 7 & 4 & \\
\hline Necrosis, $\mathrm{n}$ & & & 0.779 & & & 0.394 & & & 0.646 \\
\hline No & 153 & 128 & & 128 & 85 & & 25 & 43 & \\
\hline Yes & 15 & 14 & & 12 & 5 & & 3 & 9 & \\
\hline T Stage, $\mathrm{n}$ & & & 0.003 & & & 0.260 & & & $<0.001$ \\
\hline $\mathrm{T} 1-\mathrm{T} 2$ & 98 & 106 & & 83 & 60 & & 15 & 46 & \\
\hline T3-T4 & 70 & 36 & & 57 & 30 & & 13 & 6 & \\
\hline N Stage, $\mathrm{n}$ & & & 0.140 & & & 0.349 & & & 0.580 \\
\hline No/Nx & 159 & 139 & & 132 & 88 & & 27 & 51 & \\
\hline N1-N2 & 9 & 3 & & 8 & 2 & & 1 & 1 & \\
\hline M Stage, $\mathrm{n}$ & & & 0.002 & & & 0.024 & & & 0.018 \\
\hline $\mathrm{M} 0 / \mathrm{Mx}$ & 149 & 139 & & 126 & 88 & & 23 & 51 & \\
\hline M1 & 19 & 3 & & 14 & 2 & & 5 & 1 & \\
\hline Clinical Stage, $\mathrm{n}$ & & & 0.001 & & & 0.121 & & & $<0.001$ \\
\hline $1-2$ & 94 & 106 & & 79 & 60 & & 15 & 46 & \\
\hline $3-4$ & 74 & 36 & & 61 & 30 & & 13 & 6 & \\
\hline Furhman Grade, n & & & 0.094 & & & 0.479 & & & 0.602 \\
\hline $1-2$ & 108 & 78 & & 95 & 57 & & 13 & 21 & \\
\hline $3-4$ & 60 & 64 & & 45 & 33 & & 15 & 31 & \\
\hline
\end{tabular}

Table 3. Cox univariate and multivariate analyses of overall survival among 310 RCC patients.

\begin{tabular}{|c|c|c|c|c|c|c|}
\hline \multirow{2}{*}{$\begin{array}{l}\text { RCC patients }(\mathrm{n}= \\
310)\end{array}$} & \multicolumn{3}{|c|}{ Univariate analysis } & \multicolumn{3}{|c|}{ Multivariate analysis } \\
\hline & HR & $95 \% \mathrm{CI}$ & $\begin{array}{l}p \\
\text { value }\end{array}$ & HR & $95 \% \mathrm{CI}$ & $\begin{array}{l}p \\
\text { value }\end{array}$ \\
\hline ender (F/M) & 082 & $0.760-1.539$ & 0.662 & & - & - \\
\hline ge $(y$ & & $1.027-1.059$ & $\begin{array}{l}< \\
0.001\end{array}$ & 1.043 & $1.027-1.059$ & $\begin{array}{l}< \\
0.001\end{array}$ \\
\hline Гumor Size (cm) & 1.104 & $1.060-1.150$ & $\begin{array}{l}< \\
0.001\end{array}$ & 1.020 & $0.966-1.078$ & 0.477 \\
\hline $\begin{array}{l}\text { apsu } \\
\text { es/1 }\end{array}$ & 3.187 & $2.260-4.494$ & $\begin{array}{l}< \\
0.001\end{array}$ & 1.745 & & 0.029 \\
\hline Vein & 2.840 & $1.958-4.120$ & $\begin{array}{l}< \\
0.001\end{array}$ & 1.364 & & 0.268 \\
\hline & 588 & 0.94 & 0.083 & - & - & - \\
\hline $\begin{array}{l}\text { T Sta } \\
\text { T1-T2 }\end{array}$ & 3.368 & $2.396-4.734$ & $\begin{array}{l}< \\
0.001\end{array}$ & 1.504 & $0.497-3.271$ & 0.760 \\
\hline $\begin{array}{l}\text { N Stage (N1-N2 vs. } \\
\text { N0/Nx) }\end{array}$ & 4.914 & $2.626-9.198$ & $\begin{array}{l}< \\
0.001\end{array}$ & 3.110 & & 0.002 \\
\hline $\begin{array}{l}\text { M Stage (M1 vs. } \\
\text { M0/Mx) }\end{array}$ & 4.224 & $2.584-6.904$ & $\begin{array}{l}< \\
0.001\end{array}$ & 1.537 & $0.670-3.523$ & 0.310 \\
\hline Clinical Stage & 1.783 & $1.526-2.082$ & $\begin{array}{l}< \\
0.001\end{array}$ & 1.334 & $1.050-1.695$ & 0.018 \\
\hline Furhman Grade & 1.475 & $1.228-1.771$ & $\begin{array}{l}< \\
0.001\end{array}$ & 1.508 & $1.238-1.837$ & $\begin{array}{l}< \\
0.001\end{array}$ \\
\hline nme (nign/low) & 0.624 & $0.441-0.884$ & 0.008 & 0.657 & $0.455-0.949$ & 0.025 \\
\hline
\end{tabular}

The effect of $5 \mathrm{hmC}$ in prognosis of $\mathrm{ccRCC}$, the major subtype of RCC, were also of interest. Univariate and multivariate survival analyses revealed that age (HR: 1.042; 95\%CI: 1.023-1.061; $\mathrm{p}<0.001$ ), $\mathrm{N}$ stage (HR: 2.176; 95\%CI: 0.989-4.786; $\mathrm{p}=0.053$ ), clinical stage (HR: 1.537; 95\%CI: 1.257-1.880; $\mathrm{p}<0.001$ ) and Furhman grade (HR: 1.349; 95\%CI: 1.068-1.703; $p=0.012$ ) were independent prognostic factors for ccRCC patient OS, but vein invasion (HR: 2.273; 95\%CI: 1.187-4.351; $\mathrm{p}=0.013$ ), clinical stage (HR: 1.940; 95\%CI: 1.385-2.718; p<0.001), Furhman grade (HR: 1.661; 95\%CI: 1.179-2.339; $\mathrm{p}=0.004)$ and $5 \mathrm{hmC}$ level (HR: 0.381; 95\%CI: 0.188-0.774; $\mathrm{p}=0.008$ ) were the independent prognostic factors for CCRCC patient CSS (Table 5, Table 6).

These results suggested that $5 \mathrm{hmC}$ level could be considered as an important prognostic biomarker for both RCC and ccRCC. 
A (i)

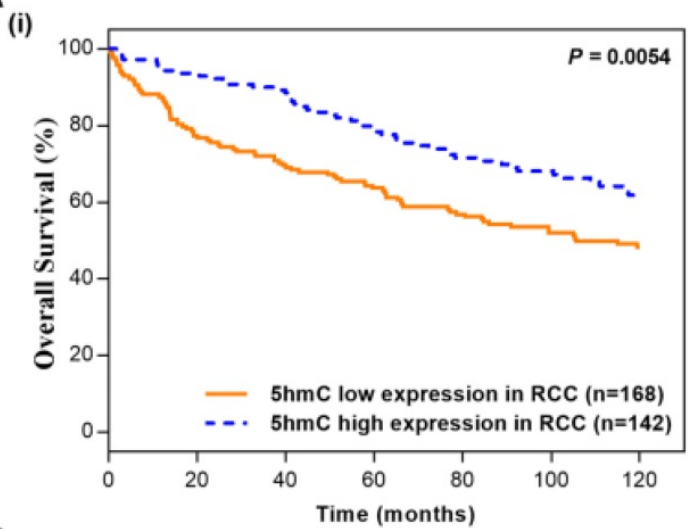

B (i)

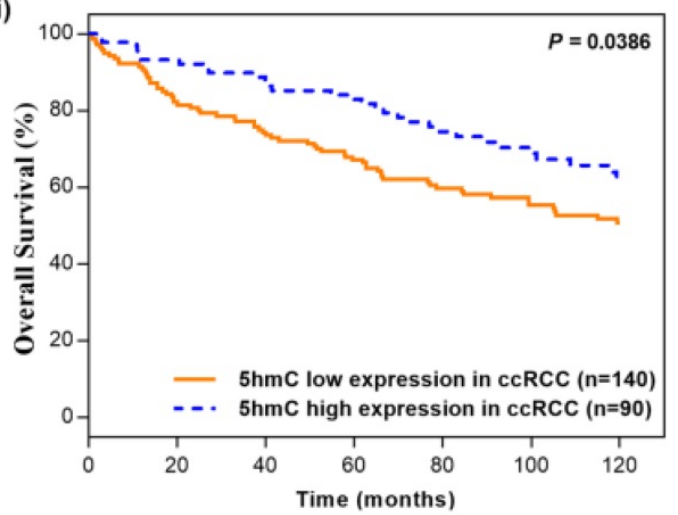

$\mathrm{C}_{\text {(i) }}$

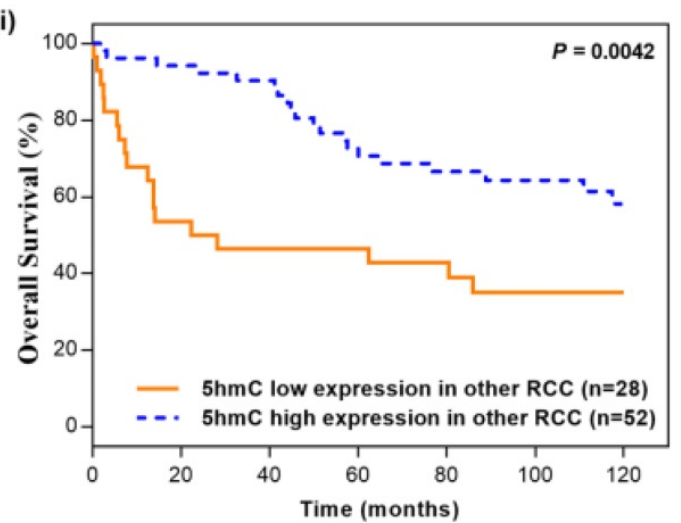

(ii)

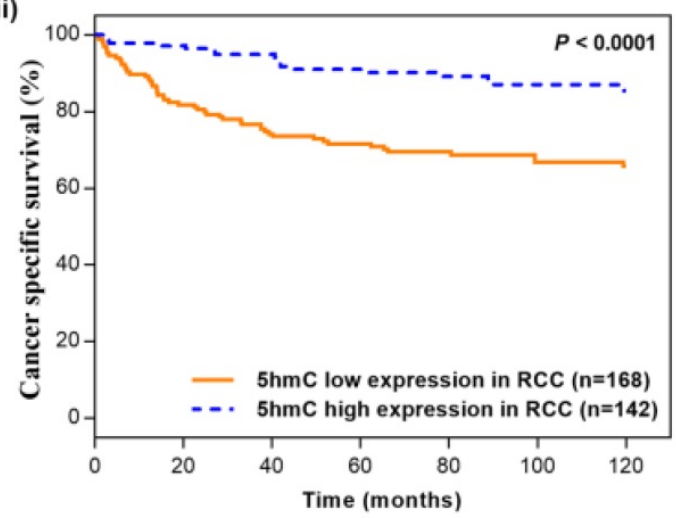

(ii)

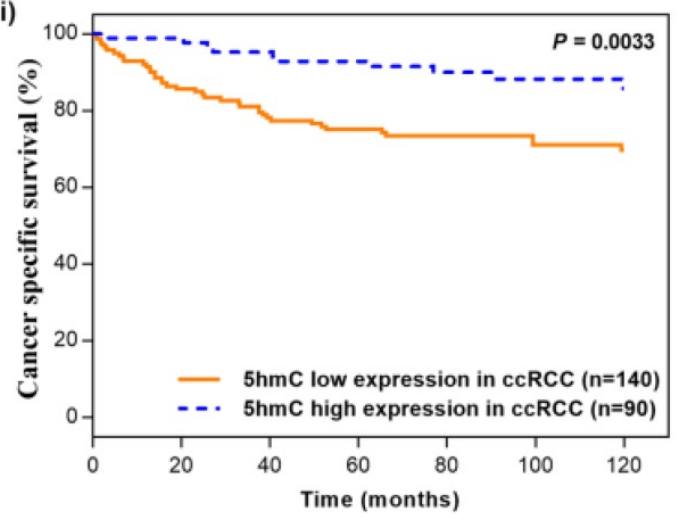

(ii)

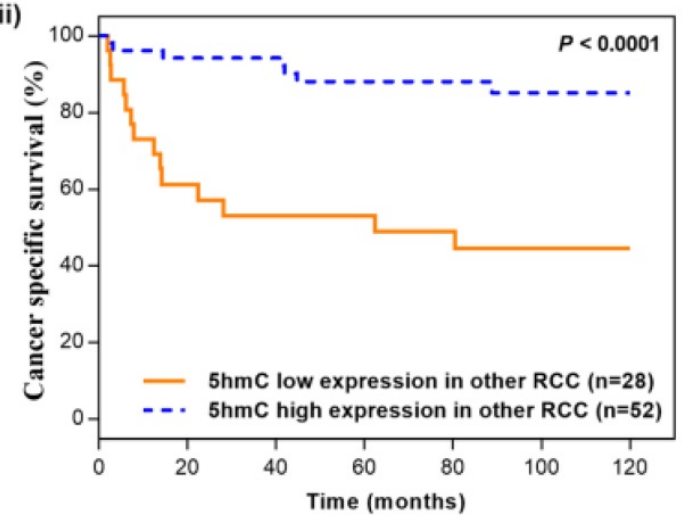

Figure 2. Kaplan-Meier survival (OS and CSS) curves of RCC patients (A) RCC patients; (B) ccRCC patients; (C) other RCC patients. "5hmC low" and "5hmC high" represented cases in which $\leq 20 \%$ and $>20 \%$ cells were positive for $\mathrm{IHC}$ staining of $5 \mathrm{hmC}$, respectively. $P$ values were calculated with the log-rank test.

Table 4. Cox univariate and multivariate analyses of cancer specific survival among 310 RCC patients.

\begin{tabular}{|c|c|c|c|c|c|c|}
\hline \multirow[t]{2}{*}{ RCC patients $(n=310)$} & \multicolumn{3}{|c|}{ Univariate analysis } & \multicolumn{3}{|c|}{ Multivariate analysis } \\
\hline & HR & $95 \% \mathrm{CI}$ & $p$ value & HR & $95 \% \mathrm{CI}$ & $p$ value \\
\hline Gender (F/M) & 1.069 & $0.657-1.740$ & 0.788 & - & - & - \\
\hline Age (years) & 1.013 & $0.995-1.032$ & 0.163 & - & - & - \\
\hline Tumor Size $(\mathrm{cm})$ & 1.166 & $1.111-1.224$ & $<0.001$ & 1.002 & $0.935-1.073$ & 0.961 \\
\hline Capsule Invasion (yes/no) & 5.553 & $3.449-8.941$ & $<0.001$ & 1.527 & $0.706-3.302$ & 0.282 \\
\hline Vein Invasion (yes/no) & 5.662 & $3.543-9.049$ & $<0.001$ & 1.427 & $0.736-2.764$ & 0.292 \\
\hline Necrosis (yes/no) & 1.571 & $0.779-3.165$ & 0.207 & - & - & - \\
\hline T Stage (T3-T4 vs. T1-T2) & 7.577 & $4.469-12.847$ & $<0.001$ & 1.736 & $0.339-8.883$ & 0.508 \\
\hline N Stage (N1-N2 vs. N0/Nx) & 7.195 & $3.650-14.184$ & $<0.001$ & 2.077 & $0.957-4.508$ & 0.064 \\
\hline M Stage (M1 vs. M0/Mx) & 6.954 & $4.001-12.088$ & $<0.001$ & 1.247 & $0.460-3.380$ & 0.665 \\
\hline Clinical Stage & 2.693 & $2.138-3.392$ & $<0.001$ & 2.381 & $1.892-2.996$ & $<0.001$ \\
\hline Furhman Grade & 1.708 & $1.328-2.197$ & $<0.001$ & 1.740 & $1.325-2.285$ & $<0.001$ \\
\hline $5 \mathrm{hmC}$ (high/low) & 0.355 & $0.208-0.606$ & $<0.001$ & 0.404 & $0.231-0.709$ & 0.002 \\
\hline
\end{tabular}


Table 5. Cox univariate and multivariate analyses of overall survival among 230 ccRCC patients.

\begin{tabular}{|c|c|c|c|c|c|c|}
\hline \multirow[t]{2}{*}{ ccRCC patients $(n=230)$} & \multicolumn{3}{|c|}{ Univariate analysis } & \multicolumn{3}{|c|}{ Multivariate analysis } \\
\hline & HR & $95 \% \mathrm{CI}$ & $p$ value & $\mathrm{HR}$ & $95 \% \mathrm{CI}$ & $p$ value \\
\hline Gender (F/M) & 1.058 & $0.702-1.594$ & 0.788 & - & - & - \\
\hline Age (years) & 1.044 & $1.026-1.063$ & $<0.001$ & 1.042 & $1.023-1.061$ & $<0.001$ \\
\hline Tumor Size $(\mathrm{cm})$ & 1.092 & $1.039-1.148$ & 0.001 & 1.016 & $0.930-1.366$ & 0.900 \\
\hline Capsule Invasion (yes/no) & 2.886 & $1.928-4.320$ & $<0.001$ & 1.723 & $0.865-4.168$ & 0.274 \\
\hline Vein Invasion (yes/no) & 2.747 & $1.788-4.221$ & $<0.001$ & 1.700 & $0.899-3.217$ & 0.103 \\
\hline Necrosis (yes/no) & 2.010 & $1.040-3.885$ & 0.038 & 1.153 & $0.584-2.276$ & 0.682 \\
\hline T Stage (T3-T4 vs. T1-T2) & 3.070 & $2.052-4.593$ & $<0.001$ & 1.246 & $0.790-3.016$ & 0.282 \\
\hline N Stage (N1-N2 vs. N0/Nx) & 5.112 & $2.543-10.276$ & $<0.001$ & 2.176 & $0.989-4.786$ & 0.053 \\
\hline M Stage (M1 vs. M0/Mx) & 3.886 & $2.155-7.009$ & $<0.001$ & 1.327 & $0.478-3.686$ & 0.587 \\
\hline Clinical Stage & 1.749 & $1.450-2.109$ & $<0.001$ & 1.537 & $1.257-1.880$ & $<0.001$ \\
\hline Furhman Grade & 1.456 & $1.167-1.816$ & 0.001 & 1.349 & $1.068-1.703$ & 0.012 \\
\hline 5hmC (high/low) & 0.658 & $0.430-1.009$ & 0.055 & 0.724 & $0.462-1.133$ & 0.158 \\
\hline
\end{tabular}

Table 6. Cox univariate and multivariate analyses of cancer specific survival among 230 ccRCC patients.

\begin{tabular}{|c|c|c|c|c|c|c|}
\hline \multirow[t]{2}{*}{ ccRCC patients $(n=230)$} & \multicolumn{3}{|c|}{ Univariate analysis } & \multicolumn{3}{|c|}{ Multivariate analysis } \\
\hline & HR & $95 \% \mathrm{CI}$ & $p$ value & HR & $95 \% \mathrm{CI}$ & $p$ value \\
\hline Gender (F/M) & 1.228 & $0.684-2.204$ & 0.493 & - & - & - \\
\hline Age (years) & 1.012 & $0.991-1.034$ & 0.269 & - & - & - \\
\hline Tumor Size $(\mathrm{cm})$ & 1.142 & $1.074-1.214$ & $<0.001$ & 1.072 & $0.895-1.455$ & 0.497 \\
\hline Capsule Invasion (yes/no) & 4.166 & $2.377-7.302$ & $<0.001$ & 1.390 & $0.914-4.633$ & 0.146 \\
\hline Vein Invasion (yes/no) & 5.811 & $3.326-10.155$ & $<0.001$ & 2.273 & $1.187-4.351$ & 0.013 \\
\hline Necrosis (yes/no) & 1.680 & $0.665-4.247$ & 0.273 & - & - & - \\
\hline T Stage (T3-T4 vs. T1-T2) & 5.735 & $3.086-10.658$ & $<0.001$ & 1.163 & $0.742-3.472$ & 0.876 \\
\hline N Stage (N1-N2 vs. N0/Nx) & 7.653 & $3.544-16.525$ & $<0.001$ & 2.203 & $0.893-5.434$ & 0.086 \\
\hline M Stage (M1 vs. M0/Mx) & 6.206 & $3.153-12.216$ & $<0.001$ & 2.129 & $0.645-7.026$ & 0.215 \\
\hline Clinical Stage & 2.464 & $1.859-3.265$ & $<0.001$ & 1.940 & $1.385-2.718$ & $<0.001$ \\
\hline Furhman Grade & 1.601 & $1.183-2.169$ & 0.002 & 1.661 & $1.179-2.339$ & 0.004 \\
\hline 5hmC (high/low) & 0.405 & $0.207-0.791$ & 0.008 & 0.381 & $0.188-0.774$ & 0.008 \\
\hline
\end{tabular}

\section{Construction of nomogram to predict survival probability}

Based on our cox regression analyses, nomograms were constructed to calculate each RCC patient survival probability directly. The 10-year OS (Figure 3A) and CSS (Figure 3B) probabilities of RCC patients were able to be accurately calculated via the nomograms according to the information of each patient $(5 \mathrm{hmC}$, age, capsule invasion, $\mathrm{N}$ stage, clinical stage, Furhman grade). The calibration curves displayed good agreements of the nomogram-predicted probability with the actual probability for OS (Figure 3C) and CSS (Figure 3D), which indicated that these nomograms had a great value of prediction. Figure $4 \mathrm{~A}$ and $4 \mathrm{~B}$ were the nomograms of ccRCC patient 10 -year OS and CSS, respectively. The calibration curves shown in Figure 4C and 4D, also displayed good agreement.

\section{External validation for the prediction nomograms}

To confirm the stability of the prediction nomograms, external data validations were performed, which was independently collected in another center (TMA). For 10-year OS prediction of RCC, the sensitivity was $77.3 \%$ and the specificity was $81.8 \%$; for 10-year OS prediction of ccRCC, the sensitivity was $76.7 \%$ and the specificity was $82.8 \%$ (Supplementary Table s3-s4).

Taken together, the results well validated the main findings, the prediction nomograms exhibit high accuracy and stability and are well generalized for other independent datasets.

\section{Discussion}

As a mature epigenetic abnormal change, suppression of $5 \mathrm{mC}$ is a common DNA modification in human malignant tumors. It therefore has great potential as a kind of cancer treatment target [16, 23-24]. Accumulating evidence suggests that $5 \mathrm{hmC}$ confers unique epigenetics, and it can play an important role in multiple tumors, such as liver cancer, melanoma, and acute myeloid leukemia [25-28]. In this study, both dot blot and IHC indicated that $5 \mathrm{hmC}$ was significantly decreased in RCC compared with those in their normal tissue counterparts. Kaplan-Meier curves revealed that high $5 \mathrm{hmC}$ level led to good prognostic impact for RCC patients. Our study established $5 \mathrm{hmC}$ loss as a probably common epigenetic characteristic in human tumors including RCC, as with the other reports [25-28]. For this reason, we proposed that $5 \mathrm{hmC}$ loss might correlated with kidney tumorigenesis and then investigated. 
A

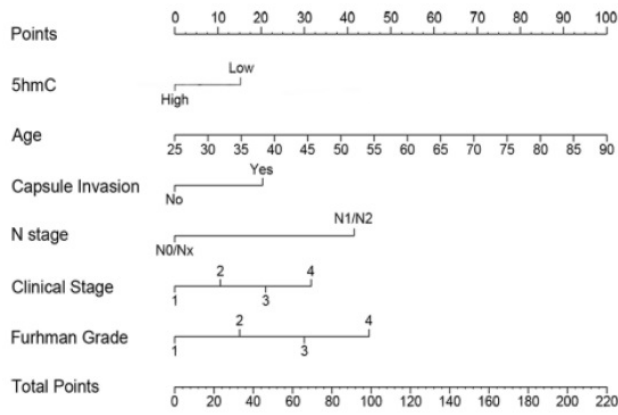

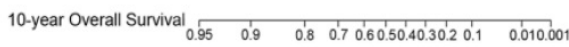

C

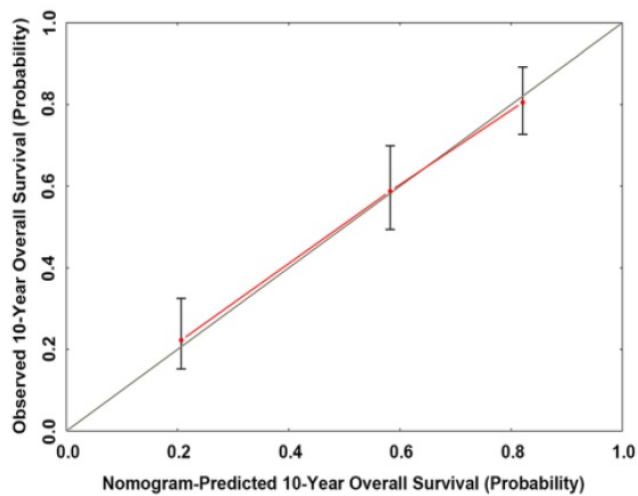

Points

$5 \mathrm{hmC}$

Clinical Stage

Furhman Grade

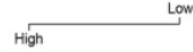

Total Points

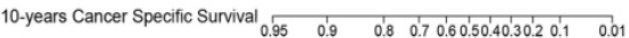

D

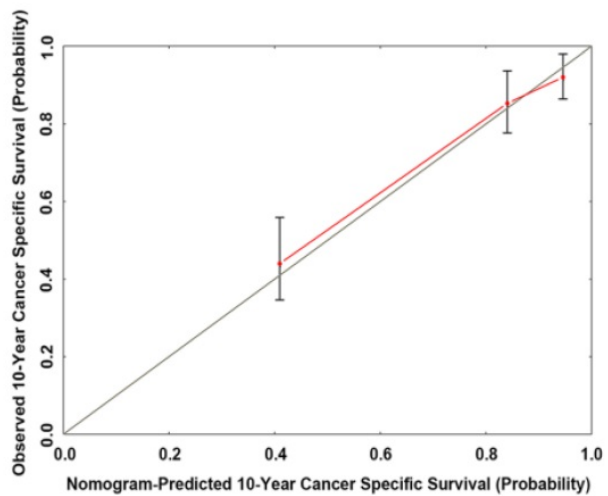

Figure 3. The predicted nomograms and calibration curves of RCC patient survival (A) the nomogram developed for OS among 310 RCC patients; (B) the nomogram developed for CSS among 310 RCC patients; (C) The calibration curve developed for OS among 310 RCC patients; (D) The calibration curve developed for CSS among 310 RCC patients. For the nomogram, the points for each variable were calculated by drawing a straight line from a variable value to the axis labelled "Points". The score sum was converted to a probability in the lowest axis.

A

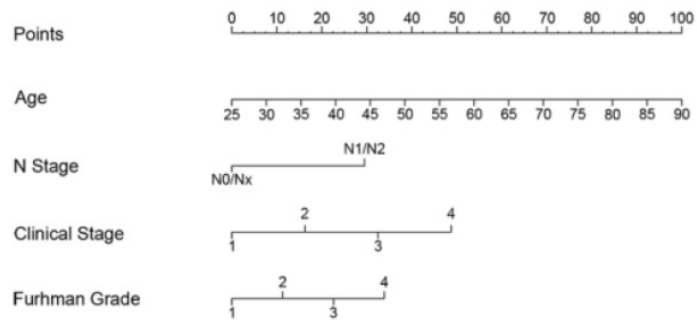

Total Points

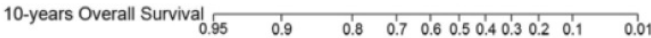

C

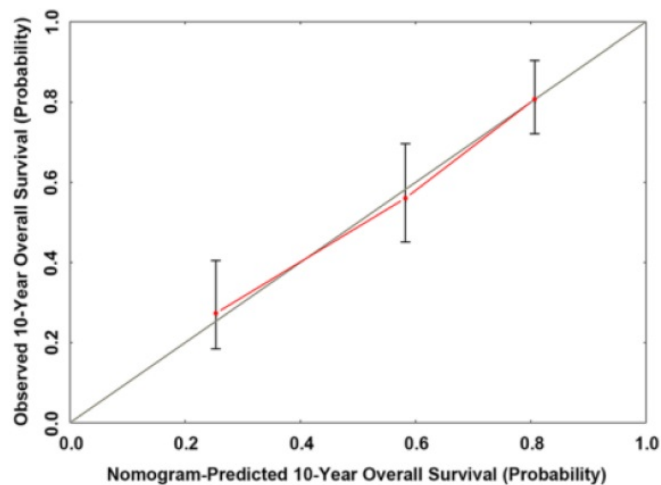

Points

$5 \mathrm{hmc}$

Vein Invasion

Clinical Stage

Furhman Grade

Total Points

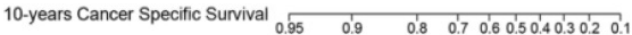

D

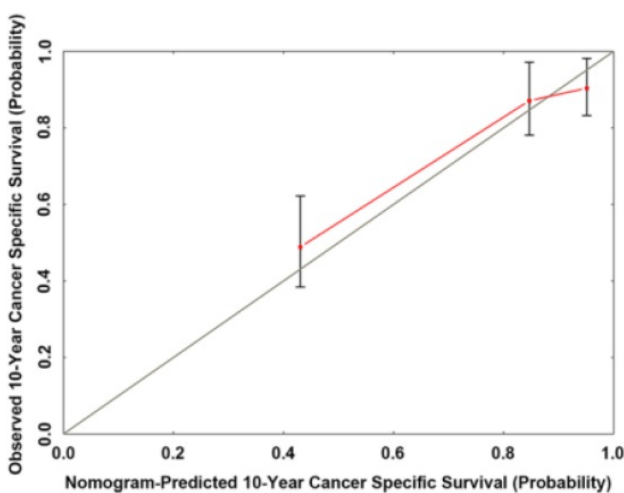

Figure 4. The predicted nomograms and calibration curves of ccRCC patient survival (A) The nomogram developed of OS among 230 ccRCC patients; (B) The nomogram developed for CSS among 230 ccRCC patients; (C) The calibration curve developed for OS among 230 ccRCC patients; (D) The calibration curve developed for CSS among 230 ccRCC patients. In the nomogram, the points for each variable were calculated by drawing a straight line from a patient's variable value to the axis labelled "Points". The score sum was converted to a probability in the lowest axis. 
Numerous studies have shown that the loss of $5 \mathrm{hmC}$ is associated with the cancer aggressiveness [25-26]. In gastric cancer, a decrease in $5 \mathrm{hmC}$ promoted the metastasis of gastric cancer cells. Furthermore, suppression of $5 \mathrm{hmC}$ was more likely to be present in high grade pathological and large volume tumors in gliomas. In the melanoma, glioma, and esophageal squamous cell carcinoma, the reduction of $5 \mathrm{hmC}$ was shown to be an epigenetic hallmark [29-31]. However, the prognostic value of $5 \mathrm{hmC}$ in renal cell carcinoma (RCC) is still unclear.

This study indicated that low $5 \mathrm{hmC}$ level was significantly associated with tumor size, capsule invasion, vein invasion, $\mathrm{T}$ stage, $\mathrm{M}$ stage, clinical stage, shorter OS and CSS in our RCC patient cohort. Furthermore, Kaplan-Meier curves revealed that the high $5 \mathrm{hmC}$ expression had a good prognostic impact in RCC patients. More importantly, cox univariate and multivariate survival analyses further indicated $5 \mathrm{hmC}$ was independent prognostic factor for RCC affecting patient survival. These findings suggested that $5 \mathrm{hmC}$ might have great values of prognostic in RCC patients.

In recent years, nomogram has been widely used in clinical research modeling. It transforms the complex cox regression survival analyses into a simple and visualized graph, which makes the results of the prediction model more readable and has higher application value. The advantage makes the nomograms get more attention and application in medical research and clinical practice. With the 10-year follow-up data of 310 RCC patients, we developed nomograms to calculate RCC survival probability for the first time. The calibration curves displayed good agreements, which showed nomograms had a great value of prediction. The nomograms may be used to calculate the 10-year OS and CSS probabilities of RCC patients accurately according to the information of each patient. Therefore, clinicians can use these nomograms to make the treatment planning and patient-clinician communication. In addition, our findings were validated with an independent external cohort included 77 RCC cases. The prediction nomograms exhibit high accuracy and stability and are well generalized for other independent datasets.

Of course, considering the effect of racial/ethnic differences and the limitation of small amount of data, multiple center data and more cases are needed for further study.

\section{Conclusion}

$5 \mathrm{hmC}$ was significantly downregulated in RCC patients. The level of $5 \mathrm{hmC}$ was significantly correlated with capsule invasion, vein invasion, $\mathrm{T}$ stage, $\mathrm{M}$ stage and clinical stage. High $5 \mathrm{hmC}$ level led to good prognostic impact for RCC patients and $5 \mathrm{hmC}$ was independent prognostic factor in RCC patient survival. In order to establish $5 \mathrm{hmC}$ as a novel biomarker for RCC in future, more investigations of its expression, function and regulation are warranted.

\section{Abbreviations}

AUC: Areas Under the Curve; ccRCC: Clear Cell Renal Cell Carcinoma; index: Concordance Index; CSS: Cancer Specific Survival; FBS: Fetal Bovine Serum; 5hmC: 5-hydroxymethylcytosine; 5mC: DNA Methylation of Cytosine; IDH1/2: Isocitrate Dehydrogenase 1 and 2; IHC: Immunohistochemical; OS: Overall Survival; RCC: Renal Cell Carcinoma; ROC: Receiver Operating Characteristic; RT: Room Temperature; TET: Ten-Eleven-Translocation; VHL: Von Hippel-Lindau.

\section{Supplementary Material}

Supplementary figures and tables. http://www.jcancer.org/v11p1212s1.pdf

\section{Acknowledgements}

We would like to acknowledge the excellent technical assistance of Yuan Zhu and Danni Shan. The funders had no role in study design, data collection and analysis, decision to publish, or preparation of the manuscript.

\section{Funding}

This study was funded by the National Natural Science Foundation of China (grant number: 81702513) and Zhongnan Hospital of Wuhan University Science, Technology and Innovation Seed Fund Project (grant number: znpy2016018).

\section{Ethical approval}

All procedures performed in studies involving human participants were in accordance with the ethical standards of the institutional and/or national research committee and with the 1964 Helsinki declaration and its later amendments or comparable ethical standards.

\section{Informed consent}

Informed consent was obtained from all individual participants included in the study.

\section{Data Availability Statement}

The data that support the findings of this study are available from the corresponding author upon reasonable request. 


\section{Competing Interests}

The authors have declared that no competing interest exists.

\section{References}

1. Bray F, Ferlay J, Soerjomataram I, Siegel RL, Torre LA, Jemal A. Global Cancer Statistics 2018: GLOBOCAN Estimates of Incidence and Mortality Worldwide for 36 Cancers in 185 Countries. CA Cancer J Clin. 2018; Sep 12.

2. Jemal A, Bray F, Center MM, Ferlay J, Ward E, Forman D. Global cancer statistics. CA Cancer J Clin. 2011; 61: 69-90.

3. Petrozza V, Carbone A, Bellissimo T, et a1. Oncogenic MicroRNAs Characterization in Clear Cell Renal Cell Carcinoma. Int J Mol Sci. 2015; 16: 29219-25.

4. Jonasch E, Futreal PA, Davis IJ, et a1. State of the science: an update on renal cell carcinoma. Mol Cancer Res. 2012; 10: 859-80.

5. Motzer RJ, Bander NH, Nanus DM: Renal-cell carcinoma. N Engl J Med. 1996; 335: 865-875

6. Kim HL, Halabi S, Li P, et a1. A Molecular Model for Predicting Overall Survival in Patients with Metastatic Clear Cell Renal Carcinoma: Results from CALGB 90206 (Alliance). EBioMedicine. 2015; 2: 1814-20.

7. Escudier B, Eisen T, Stadler WM, Szczylik C, Oudard S, Siebels M, et a1. Sorafenib in advanced clear-cell renal-cell carcinoma. N Engl J Med. 2007; 356: 125-34.

8. Mubarak M, Kazi JI, Mohsin R, et a1. Histopathology of surgically treated renal tumors in young adults: a developing country perspective. Cancer Res Clin Oncol. 2012; 138: 189-94.

9. Fu Q, Chang Y, An H, Fu H, Zhu Y, Xu L, et al. Prognostic value of interleukin-6 and interleukin-6 receptor in organ-confined clear-cell renal cell carcinoma: a 5-year conditional cancer-specific survival analysis. Br J Cancer. 2015; 113: 1581-9.

10. Dall'Oglio MF, Antunes AA, Sarkis AS, Crippa A, Leite KR, Lucon AM, Srougi M. Microvascular tumour invasion in renal cell carcinoma: the most important prognostic factor. BJU Int. 2007; 100: 552-5.

11. Bedke J, Heide J, Ribback S, Rausch S, de Martino M, Scharpf M, et al. Microvascular and lymphovascular tumour invasion are associated with poor prognosis and metastatic spread in renal cell carcinoma: a validation study in clinical practice. BJU Int. 2018; 121: 84-92.

12. Kim WY, Kaelin WG. Role of VHL gene mutation in human cancer. J Clin Oncol. 2004; 22: 4991-5004.

13. Kapitsinou PP, Haase VH. The vhl tumor suppressor and HIF: insights from genetic studies in mice. Cell Death Differ. 2008; 15: 650-9.

14. Creighton CJ, Morgan M, Gunaratne $\mathrm{PH}$, et al. Comprehensive molecular characterization of clearcell renal cell carcinoma. Nature. 2013; 499: 43-9.

15. Yusuke S, Tetsuichi $Y$, Yuichi S, et al. Integrated molecular analysis of clear-cell renal cell carcinoma. Nat Genet. 2013; 45: 860-7.

16. Ha PK, Califano JA. Promoter methylation and inactivation of tumour-suppressor genes in oral squamous-cell carcinoma. Lancet Oncol. 2016; 7: 77-82.

17. Rasmussen KD, Helin K. Role of TET enzymes in DNA methylation, development, and cancer. Genes Dev. 2016; 30: 733-50.

18. Yang J, Guo R, Wang H, Ye X, Zhou Z, Dan J, et al. Tet Enzymes Regulate Telomere Maintenance and Chromosomal Stability of Mouse ESCs. Cell Rep. 2016; 15: 1809-21.

19. Liu M1, Ohtani H1, Zhou W1, Ørskov AD2, Charlet J3, Zhang YW, et al. Vitamin C increases viral mimicry induced by 5-aza-2'-deoxycytidine. Proc Natl Acad Sci U S A. 2016; 113: 10238-44.

20. Nestor CE, Ottaviano R, Reddington J, Sproul D, Reinhardt D, Dunican D, et a1. Tissue type is a major modifier of the 5-hydroxymethylcytosine content of human genes. Genome Res. 2012; 22: 467-77.

21. Branco MR, Ficz G, Reik W. Uncovering the role of 5-hydroxymethylcytosine in the epigenome, Nat Rev Genet. 2012; 13: 7-13.

22. Chen K, Zhang J, Guo Z, Ma Q, Xu Z, Zhou Y, et a1. Loss of 5-hydroxymethylcytosine is linked to gene body hypermethylation in kidney cancer. Cell Res. 2016; 26: 103-18.

23. Dawson MA, Kouzarides T. Cancer epigenetics: from mechanism to therapy. Cell. 2012; 150: 12-27.

24. Campbell RM, Tummino PJ. Cancer epigenetics drug discovery and development: the challenge of hitting the mark. J Clin Invest. 2014; 124: 64-9.

25. Ficz G, Gribben JG. Loss of 5-hydroxymethylcytosine in cancer: cause or consequence? Genomics. 2014; 104: 352-7.

26. Jin SG, Jiang Y, Qiu R, Rauch TA, Wang Y, Schackert G, et a1. 5-Hydroxymethylcytosine is strongly depleted in human cancers but its levels do not correlate with IDH1 mutations. Cancer Res. 2011; 71: 7360-5.

27. Lu F, Liu Y, Jiang L, Yamaguchi S, Zhang Y. Role of Tet proteins in enhancer activity and telomere elongation. Genes Dev. 2014; 28: 2103-19.

28. Thomson JP, Ottaviano $R$, Unterberger EB, Lempiäinen $H$, Muller A, Terranova R, et a1. Loss of Tet1-Associated 5-Hydroxymethylcytosine Is Concomitant with Aberrant Promoter Hypermethylation in Liver Cancer. Cancer Res. 2016; 76: 3097-108.
29. Zhang F, Liu Y, Zhang Z, Li J, Wan Y, Zhang L, et a1. 5-hydroxymethylcytosine loss is associated with poor prognosis for patients with WHO grade II diffuse astrocytomas. Sci Rep. 2016; 6: 20882.

30. Lian CG, Xu Y, Ceol C, Wu F, Larson A, Dresser K, et a1. Loss of 5-hydroxymethylcytosine is an epigenetic hallmark of melanoma. Cell. 2012; 150: $1135-46$.

31. Shi X, Yu Y, Luo M, Zhang Z, Shi S, Feng X, et a1. Loss of 5-Hydroxymethylcytosine Is an Independent Unfavorable Prognostic Factor for Esophageal Squamous Cell Carcinoma. PLoS One. 2016; 11: e0153100. 